\title{
SELF-SIMILAR BLOWUP SOLUTIONS TO AN AGGREGATION EQUATION IN $R^{N}$
}

\author{
YANGHONG HUANG* AND ANDREA L. BERTOZZI*
}

\begin{abstract}
We present numerical simulations of radially symmetric finite time blowup for the aggregation equation $u_{t}=\nabla \cdot(u \nabla K * u)$, where the kernel $K(x)=|x|$. The dynamics of the blowup exhibits self-similar behavior in which zero mass concentrates at the core at the blowup time. Computations are performed in $R^{n}$ for $n$ between 2 and 10 using a method based on characteristics. In all cases studied, the self-similarity exhibits second kind (anomalous) scaling.
\end{abstract}

Key words. aggregation equation, self-similarity solution of the second kind, blowup

AMS subject classifications. 74H15, 74H35, 76M55, 82B24

1. Introduction. In this paper, we consider the self-similar blowup of radially symmetric solutions to the aggregation equation

$$
u_{t}=\nabla \cdot(u \nabla K * u) \quad \text { in }[0, T) \times \mathbb{R}^{n},
$$

subject to initial condition $u(0, x)=u_{0}(x)$ on some time interval of existence $[0, T)$. Here $K * u$ is the convolution of $u$ with a radially symmetric kernel potential $K$. This equation arises in a number of continuum models for interacting particles via pairwise potentials. It appears in the description of self-assembly of nanoparticles by Holm and Putkaradze [32, 33] as an alternative to the Debye-Huckel or Keller-Segel model. It is also used as a popular model for aggregation in animal behavior, the schools or swarms formed by fish and birds, where everyone senses the presence of other individuals $[12,19,20,44,49,48]$. In these applications the most common potential in the literature is $K(x)=1-e^{-|x|}$, which behaves like $|x|$ near the origin. These are examples of Lipschitz continuous potentials for which the lack of higher regularity at $x=0$ is known to be responsible for the finite time blowup.

The basic properties of the equation (1.1), with a 'pointy' potential as described above, are addressed recently in a series of papers. Bodnar and Velazquez [12] study the problem of existence and uniqueness, along with blowup and steady states in one dimension for different potentials with smooth initial data. The local existence and uniqueness of this equation in higher dimensions is proved by Laurent [38]. An alternative local existence and finite time blowup proof is given by Bertozzi and Laurent in [8]. The blowup problem is revisited in [7] and the review article [9] with a more general class of potentials, for which it is determined that an Osgood condition on the regularity of the potential at the origin is necessary and sufficient for finite time blowup. Moreover, it is shown that there is no mass-concentrating, smooth self-similar finite time blowup solution in odd dimension larger than one, for the homogeneous, radially symmetric kernel $K(x)=|x|$. This fact is the primary motivation for the study here namely that the mostly common multidimensional models in the literature are known to produce finite time singularities and in some higher dimensions, no smooth similarity solutions exist that concentrate mass. The paper [7] has a simple example in one dimension of a mass-conserving similarity solution. In higher dimensions they show some interesting similarity solutions that concentrate mass, but all of these involve collapsing delta-rings and thus cannot result from smooth initial data. By performing numerical calculations with a high degree of spatial and temporal precision, we are able to resolve the dynamics of blowup for this problem in multiple space dimensions. Our assumption of radial symmetry allows us to obtain a degree of precision that would be much more difficult in the case of solutions lacking symmetry.

*Department of Mathematics, UCLA, Los Angeles, CA 90095. email: \{yhhuang, bertozzi\} @math.ucla.edu 
Finite time blowup phenomena appear in many equations for physical models, including semilinear heat equations [29, 28], nonlinear Schrdinger equations [30, 42, 27], gravitational collapse [14] and pinchoff in surface diffusion [5]. For a general review article, see [26]. Near the blowup time, it often happens that, because of the absence of any external scales, the solution collapses to the singularity in a self-similar way. Probably the most extensively studied one is the semilinear heat equation

$$
u_{t}=\Delta u+f(u), \quad f(u)=u^{p} \text { or } f(u)=e^{u} .
$$

However, it has been well-known since the 1970s that there is no exact self-similar solution of the form (for $f(u)=u^{p}$ )

$$
u(x, t)=(T-t)^{-1 /(p-1)} U\left(x /(T-t)^{1 / 2}\right) .
$$

A refined analysis or center manifold theory close to the blowup time gives the following asymptotic behavior with a logarithm correction $[25,28,29,43]$

$$
u(x, t) \sim\left(\frac{\beta}{T-t}\right)^{\beta}\left(1+\frac{p-1}{4 p} \eta^{2}\right), \quad \text { where } \eta=\frac{x}{\sqrt{[(T-t)|\ln (T-t)|}}, \beta=\frac{1}{p-1} .
$$

In contrast, quasilinear problems $[47,17]$

$$
u_{t}=\left(\left|u_{x}\right|^{\sigma} u_{x}\right)_{x}+e^{u} \quad \text { or } \quad u_{t}=\left(u^{\sigma} u_{x}\right)_{x}+u^{p}, \quad \sigma>0
$$

or higher order parabolic equations [16]

$$
u_{t}=(-1)^{m+1} D_{x}^{2 m} u+|u|^{p-1} u, \quad \text { or } \quad u_{t}=(-1)^{m+1} D_{x}^{2 m} u+e^{u}
$$

do possess exact self-similar blowup solutions, where $D_{x}^{2 m}$ is the $2 \mathrm{~m}$-th derivative with respect to $x$.

For those solutions with nontrivial blowup profiles, it is possible that the profiles match the exact analytical ones only near the core of the blowup point (or set), with deviation (though very small in magnitude) away from the core, sometimes called quasi self-similar solutions. This is observed in the collapse of the cubic Nonlinear Schrodinger Equation, either for the Townes profile $[24,42,45]$ or for the ring profile [27].

In finite blowup problems for density distributions, the dynamics could concentrate a finite amount of mass or zero mass in the core, even for different types of blowup solutions of the same equation which conserves the total mass. One example is the Keller-Segel system of equations, which model overdamped gravitational interaction of a cloud of particles and chemotaxis in bacteria [37]

$$
\begin{aligned}
& \partial_{t} \rho=\Delta \rho-\nabla \cdot(\rho \nabla c), \\
& -\Delta c=\rho,
\end{aligned}
$$

where $\rho$ is the density of the cloud or the bacteria and $c$ represents the gravitational potential or the density of the chemo-attractant. In spatial dimension greater than two, there are at least two types of blowup solutions. One is exactly self-similar, concentrating zero mass, and the other is like a Burgers shock, with finite mass in a ring converging to the origin [13].

For all of the similarity solutions considered above, the similarity variables and exponents characterizing the dynamics, especially those related to the spatial spread, can be determined a priori, either from a dimensional analysis of the problem or the basic invariant scales of the equation, called self-similar solutions of the first kind or complete similarity solutions. While, other problems exist in which self-similar scaling exponents cannot be determined from 
dimensional analysis; these are the so-called similarity solutions of the second kind or incomplete similarity solutions [4]. Among the first few problems studied are the nonlinear filtration by Barenblatt [3], Vázquez and collaborators [36, 2] and Peletier [46], and the focusing problem of the porous medium equation [35, 1]. In physics, those exponents are said to be anomalous. Sometimes renormalization group theory originated from quantum field theory is applied to the above mentioned problems to find self-similar solutions of the second kind [31].

The goal of this paper is to show numerical evidence that the aggregation equation (1.1) has a family of radially symmetric, smooth self-similar blowup solutions. Those solutions appear to be exactly self-similar, concentrating zero mass in the core and of the second kind. The rest of the paper is organized as follows. The equation for the blowup profiles is derived in Section Two, together with numerical observations of the profiles and anomalous exponents in different space dimensions. In Section Three we present the numerical methods used to simulate the blowup dynamics and a numerical Renormalization Group method to find the exponents iteratively. Additional numerical results about the postprocessing of the data from the blowup dynamics and the blowup in different $L^{p}$ norms are given in section Four. We end this paper with some further directions and open questions in Section Five.

\section{Self-similar solutions of the blowup dynamics.}

2.1. General theory for the self-similar blowup profiles. We introduce the following similarity variables $y$ and $\tau$

$$
y=x(T-t)^{-\beta}, \quad \tau=-\ln (T-t),
$$

and define a new function $U(y, \tau)$ such that

$$
U(y, \tau)=(T-t)^{\alpha} u\left(y(T-t)^{\beta}, T-e^{-\tau}\right),
$$

where $T$ is the blowup time, $\alpha$ and $\beta$ are exponents characterizing the singularity when the blowup time is approached. We call the blowup dynamics self-similar if the transformed function $U$ converges to some steady state as $t \rightarrow T^{-}$, or equivalently $\tau \rightarrow \infty$ for some appropriate constants $\alpha$ and $\beta$. When (2.2) is substituted into the original evolution equation for $u$, a routine calculation gives

$$
\begin{aligned}
& (T-t)^{-\alpha-1}\left(\partial_{\tau} U+\alpha U+\beta y \cdot \nabla U\right) \\
= & (T-t)^{(n-2) \beta-2 \alpha} \nabla_{y} \cdot\left(U(y, \tau) \nabla_{y} \int_{R^{n}} K\left((y-z)(T-t)^{\beta}\right) U(z, \tau) d z\right) .
\end{aligned}
$$

Therefore, a self-similar solution exists only if we can take the factor $(T-t)^{\beta}$ out of the kernel $K$. Thus $K$ must be homogeneous, i.e., $K(\lambda x)=\lambda^{d} K(x)$ for some constant $d$ and any $\lambda$. If the function $u$ is concentrated on a small region in space, say the origin, then the kernel $K$ can often be approximated by the leading nonconstant part ( $K$ is determined up to a constant), which is homogeneous. For this reason, in the rest of this paper, we will concentrate on the case with

$$
K(x)=|x|,
$$

which is an approximation to the popular kernel $K(x)=1-e^{-|x|}$ and is shown to lead to finite time blowup $[8,7]$. Given this, then the matching of the exponents of $(T-t)$ in equation $(2.3)$ gives

$$
\alpha=(n-1) \beta+1
$$

and the equation for $U$ is

$$
\partial_{\tau} U=\nabla \cdot(U \nabla K * U)-\alpha U-\beta y \cdot \nabla U .
$$


Any exact self-similar profile $U$, if it exists, must satisfy the steady equation of (2.6), i.e.,

$$
\nabla \cdot(U \nabla K * U)-\alpha U-\beta y \cdot \nabla U=0,\left.\quad \nabla U\right|_{y=0}=0, \lim _{|y| \rightarrow \infty} U(y)=0,
$$

where $U$ has no explicit dependence on $\tau$. To completely characterize the self-similar blowup dynamics, we need one extra condition to find the exponent $\beta$. Very often this kind of information can be readily available from a dimensional analysis or scale invariance of the underlying equation, like the parabolic scaling $\beta=1 / 2$ for semilinear heat equation and Nonlinear Schrdinger equation, or $\beta=1 /(2 m)$ for higher order parabolic equations as those in (1.6). Here, if the similarity solution concentrates mass in the core of the blowup, then $\alpha=n \beta$ from mass conservation, and consequently $\beta=1$. However, numerical simulation of the blowup dynamics shows that no mass is concentrated. In fact, it is proved analytically in [7] that there is no such radially symmetric, self-similar solution in odd dimension larger than one, that concentrates mass. The argument is straightforward so we review it here. Taking $\alpha=n, \beta=1$ in (2.7), we can integrate the equation in radial coordinate $r=|y|$,

$$
-n U-r U_{r}=\frac{1}{r^{n-1}} \partial_{r}\left[r^{n-1} U \partial_{r}(K * U)\right]
$$

Multiplying both sides with $r^{n-1}$ and integrating once again, we get

$$
-r^{n} U=r^{n-1} U \partial_{r}(K * U)
$$

Assuming $U$ is nonzero, we divide by $y^{n-1} U$ and integrate up again to get the final result,

$$
-\frac{1}{2} r^{2}+C=K * U
$$

Now we recognize that in odd dimension $n$ larger than one, for the special case of $K=|x|$, applying repeated Laplacians to the right hand side of (2.8) gives $\Delta^{(n-1)} K * U=c_{n} U$, whereas the left hand side gives $\Delta^{(n-1)}\left(y^{2}+C\right)=0$. Hence we do not have a nontrivial exact similarity solution of first kind (conserving mass) in odd space dimension larger than one. A more rigorous analysis and derivation of this is discussed in Lagrangian coordinates in [7]. In particular, that paper considers more general measure-valued similarity solutions due to the fact that there are easily constructed examples that concentrate mass in finite time in general space dimension, starting for initial data of the form of a delta-ring (support of the solution concentrated on the boundary of a sphere). However, here we consider solutions with $U$, a bounded function of space. Thus it is reasonable to look for similarity solutions of the second kind, for which $\alpha$ and $\beta$ satisfy equation (2.5), which comes from the dimensional analysis of the dynamics, but may violate conservation of mass.

The nonlocal nature of the kernel $K * U$ presents a much more difficult problem, both analytically and numerically, compared to local problems as those from nonlinear diffusion equations and nonlinear Schrodinger equations. The usual techniques to tackle the equation for the self-similar profiles, like phase plane analysis and shooting methods, do not work here. Smooth self-similar blowup solutions in one dimension are considered by Bodnar and Velazquez [12] for different kernel potentials $K$. The technique used there is to introduce an auxiliary function

$$
\psi(x, t)=\int_{-\infty}^{x} u(z, t) d z .
$$

Moreover, for the special kernel $K=|x|$ considered here, the transformation (2.9) turns the equation (1.1) in one dimension into $\psi_{t}=\psi_{x}(2 \psi-c)$ with $c=\psi(\infty, t)=\int_{-\infty}^{\infty} u_{0}(z) d z$, which is a constant. Another change of variable $\phi=c-2 \psi$ gives exactly the well-known inviscid 
Burgers equation $\phi_{t}+\phi \phi_{x}=0$. For general initial condition, the finite time blowup of $u$ is equivalent to the onset of shock of $\phi$, with mass concentration and thus $\alpha=\beta=1$ as considered in [7]. However, for positive, even initial condition ( the analogue for radially symmetric case in higher dimension), the blowup exhibits a different scaling. Let the self-similar blowup solution of $\phi$ be

$$
\phi(x, t)=(T-t)^{\beta-\alpha} f\left(x(T-t)^{-\beta}\right),
$$

Here the exponents are chosen such that $u=-\phi_{x} / 2$ has the same form as (2.2). Similarly, we have $\alpha=1$ and the equation for the profile

$$
f f^{\prime}+\beta y f^{\prime}-(\beta-1) f=0 .
$$

Because of the $L^{\infty}$-contraction of the solutions to Burgers equation, $\beta$ must be equal to or greater than one. If $\beta=1$, the only nontrivial solution is $f(y)=-y$, corresponding to the previous case. Otherwise if $\beta>1$, we are looking for a power series expansion of $f$ near the origin, i.e.,

$$
f(y)=a_{1} y+a_{3} y^{3}+a_{5} y^{5}+\cdots
$$

The system of equations the coefficients must satisfy is

$$
\begin{aligned}
a_{1}^{2}+a_{1} & =0 & & O(y) \\
4 a_{1} a_{3}+(2 \beta+1) a_{3} & =0 & & O\left(y^{3}\right) \\
6 a_{1} a_{5}+3 a_{3}^{2}+(4 \beta+1) a_{5} & =0 & & O\left(y^{5}\right)
\end{aligned}
$$

If $a_{1}=0$, we have the trivial solution $f \equiv 0$. Therefore $a_{1}$ must be -1 . For generic odd initial data, $a_{3}$ is nonzero, giving the exponent $\beta=3 / 2$ and the coefficients of higher order terms are determined uniquely by $a_{3}$. Otherwise, $\beta$ is decided from the next first nonvanishing term in the series.

Actually, we can directly integrate the equation (2.11) to get an implicit algebraic equation for $f$. Multiplying both sides of the differential equation (2.11) by $f(y)^{-(2 \beta-1) /(\beta-1)}$ and taking the integration once, we get

$$
f(y)^{-\frac{1}{\beta-1}}\left(1+\frac{y}{f(y)}\right)=c_{1},
$$

for some finite constant $c_{1}$. Since above equation holds in the limit when $y \rightarrow 0, f(y)$ must be $-y+o(y)$ such that $1+y / f(y)$ vanishes at the origin. Applying the condition that the limit exist once more, we can find the next higher order term of $f(y)$ must be of the form

$$
f(y)=-y+c_{1}(-y)^{\frac{\beta}{\beta-1}}+o\left((-y)^{\frac{\beta}{\beta-1}}\right) .
$$

Therefore, the exponent $\beta$ is determined by the second non-vanishing term of the profile, which is ultimately determined by the initial condition. For generic even initial condition $u_{0}, f(y)$ is odd and the next non-vanishing term is cubic, giving $\beta /(\beta-1)=3$, or $\beta=3 / 2$. This anomalous exponent is consistent with the lower bound from numerical simulation in next section.

However, this special trick and these special solutions do not seem to carry over to higher dimensions. Unlike the nonlinear filtration problem, the exponents cannot be derived using perturbation [2] or renormalization group methods [31] from known solutions in special cases or for some "unperturbed" problems. For this reason, high resolution numerical simulations are an important tool for uncovering the detailed dynamics of the blowup in higher dimensions. We summarize our results from such simulations in the next subsection. 
2.2. Numerical observations of the blowup dynamics. Here we use the same $U$ to denote the blowup profile at different times and its final steady state, and later even the radially symmetric profile, when no confusion would arise. Moreover, it is easy to check that if $U(y)$ is a solution of above equation (2.7), so is

$$
U_{\lambda}(y)=\lambda^{n-1} U(\lambda y), \quad \lambda>0
$$

and we have a family of profiles. Without loss of generality, any blowup profile shown below is normalized according to above scaling such that $U(0)=1$.
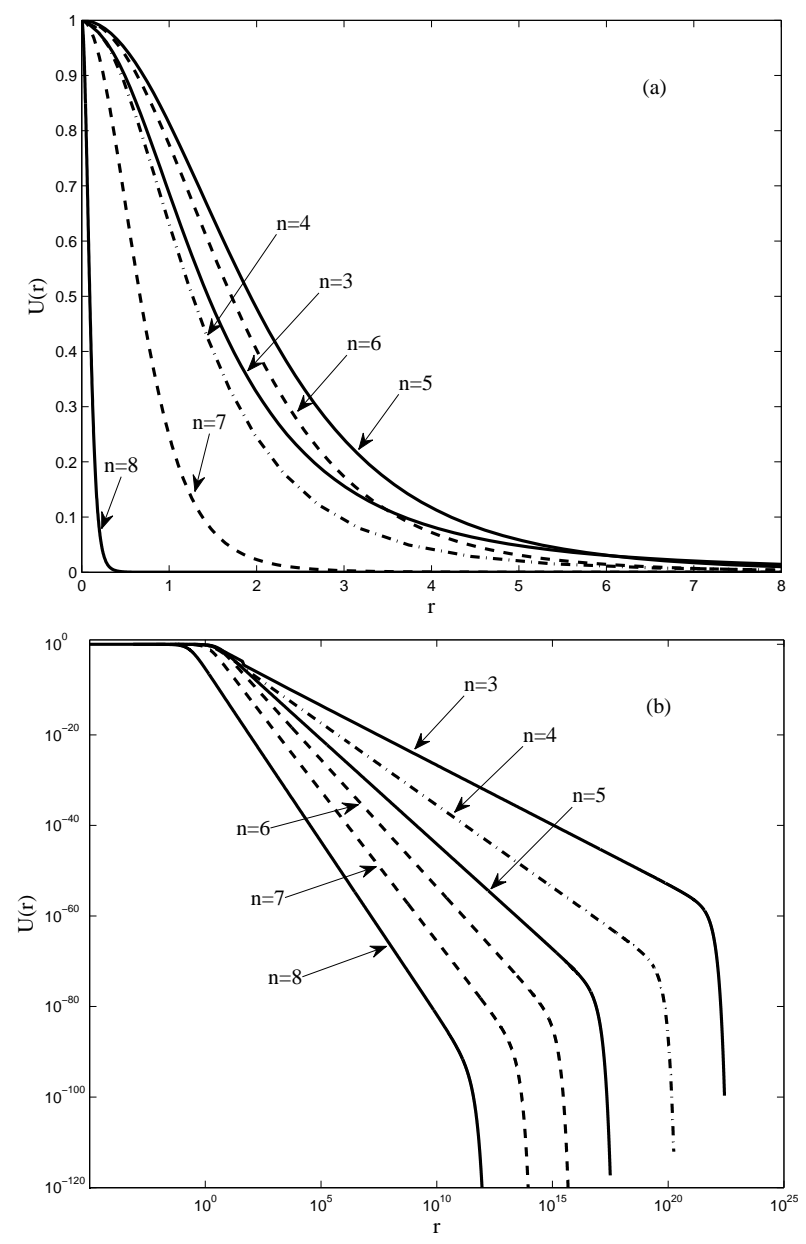

FIG. 2.1. Similarity solution profiles show in the similarity variables $U$ and $r=|y|$ as defined in (2.1-2.2), in different space dimensions, obtained by numerical integration of the PDE. All profiles are rescaled so that $U(0)=1$ according to (2.16).

Details of the numerical methods are presented later in this manuscript. The overall results show exact self-similar scaling in all dimensions studied. The normalized profiles $(U(0)=1)$ obtained from our simulations of the PDE, in different spatial dimensions, are shown in Figure 2.1. Near the origin, the profiles are not ordered according to the dimension. But far away from the origin, due to different algebraic decay rate in different dimensions, these profiles are ordered. The algebraic tails (appearing as straight lines in the right log-log plot) will extend to infinity at the blowup time. The plot on the right shows a drop-off due to the the matching of the numerical solution on to an integral order solution in the far field. Once we have the 
profiles, we can numerically check the validity of equation (2.7), which is shown in Figure 2.2 for dimension three. We observe that the part $\alpha U+\beta y \cdot \nabla U$ coming from the spatial-temporal scaling converges faster to a limit than the part associated with the kernel $\nabla \cdot(U \nabla K * U)$.

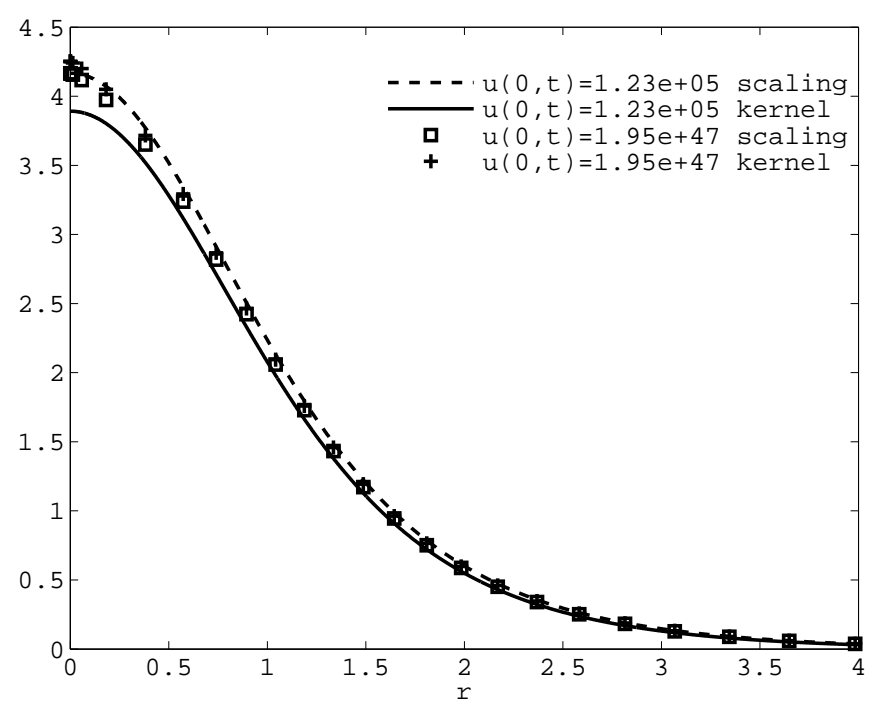

FIG. 2.2. Comparison of the two contributions $\nabla \cdot(U \nabla K * U)$ (kernel) and $\alpha U+\beta y \cdot \nabla U$ (scaling) in equation (2.7) for different $u(0, t)$ in dimension three. The term $\alpha U+\beta y \cdot \nabla U$ (dash-dots) at smaller value of $u(0, t)$ is almost indistinguishable from both terms at larger value of $u(0, t)$.

Both the exponents $\alpha$ and $\beta$ can be estimated from the simulation data (Figure 2.3, see section Four for dat fitting) . For radially symmetric solutions considered here, the computation can be extended to fractional dimension, giving more insight into the dependence of the parameters on the spatial dimension. In particular, the parameter $\beta$ appears to increase with dimension.

We can have a closer look at the detailed blowup scenario in Figure 2.4 and 2.5 for the rescaled profile $U$ and the original function $u$. Even though the results are presented only in dimension three, it is generic for all dimensions. In Figure 2.4, the rescaled profiles $U(r, \tau)$ converges to the steady state quickly near the origin and the dynamics just adjusts the algebraic decay of the tails. In Figure 2.5, the original variable $u$ is plotted at different stage during the blowup. Since the blowup takes place in such a short time, away form the core $u$ barely changes. Near the blowup point, the solution fills an envelope when approaching the blowup time. Moreover, the algebraic decay of $u$ and $U$ are intimately related through the self-similar relation (2.2). In fact any fixed $|x|>0, u(x, t)=(T-t)^{-\alpha} U\left(x(T-t)^{-\beta}, \tau\right)$ approaches a constant as $t \rightarrow T^{-}$. This gives the rate of algebraic decay for the steady profile $U, U(y) \sim$ $|y|^{-\alpha / \beta}=|y|^{-(n-1+1 / \beta)}$, making the part $\alpha U+\beta y \cdot \nabla U$ in the equation (2.7) vanish at leading order.

3. Numerical methods. The computation of blowup solutions is usually quite challenging, due to the small scale of the blowup set, which cannot be resolved quite well by conventional numerical schemes. One of the most popular schemes is the Moving Mesh Method [18, 15, 34], using an equipartition principle to give a separate equation for the mesh, to concentrate the computation on those regions where high resolution is desired. Another one is dynamic rescaling used in Nonlinear Schrodinger Equations ([42] and [27]). However, most of these schemes require a knowledge of those exponents characterizing the blowup to capture the dynamics accurately. Therefore they tend to work more successfully for self-similar solutions of the first kind. 

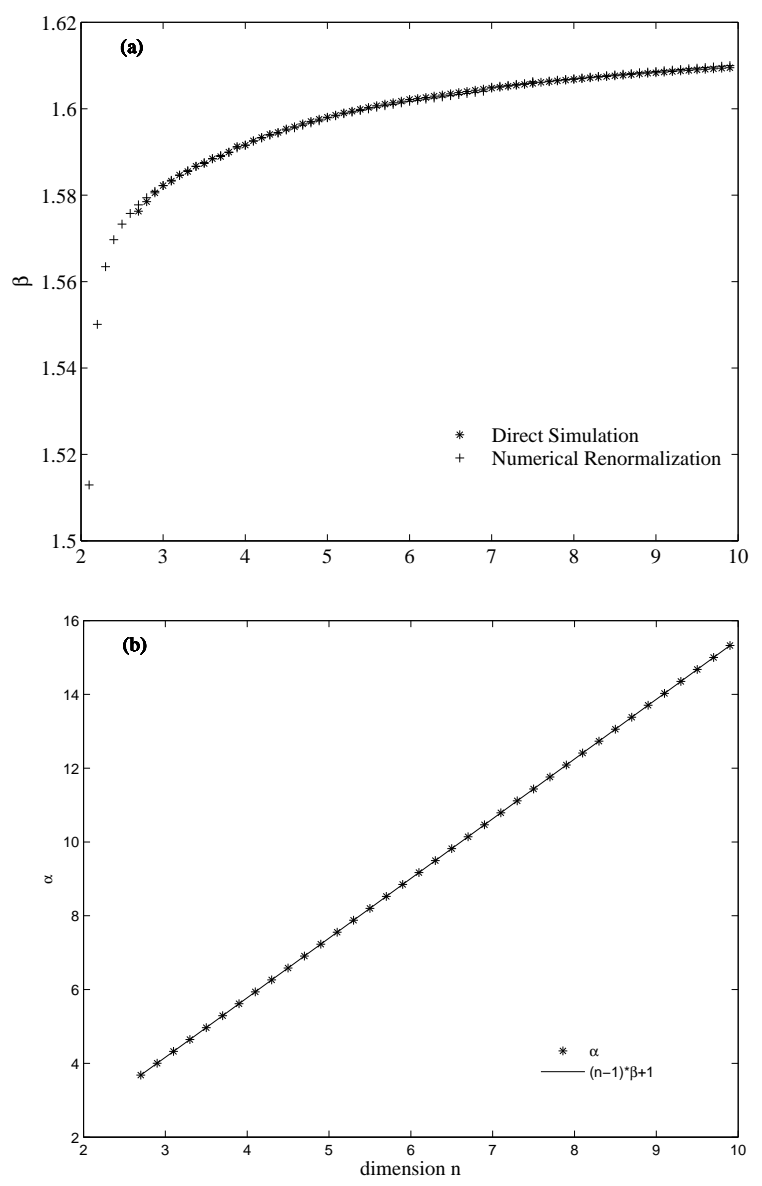

FIG. 2.3. The exponents $\beta$ and $\alpha$ characterizing the blowup in different spatial dimensions. The relation (2.5) is perfectly satisfied in the direct simulation while it is used exactly in the numerical renormalization.

Here we take advantage of the fact that our problem is a first order conservation law and thus we can use the method of characteristics to solve two coupled ODEs, one for the radial position $r$ and the other for the solution $u$. In radial coordinates, the original equation can be written as

$$
u_{t}=\frac{\partial u}{\partial r} \frac{\partial}{\partial r} K * u+u \Delta_{r} K * u,
$$

where $\Delta_{r}=\partial_{r r}+\frac{n-1}{r} \partial_{r}$. The system of ODEs along the characteristics is thus

$$
\frac{d r}{d t}=-\frac{\partial}{\partial r} K * u, \quad \frac{d u}{d t}=u \Delta_{r} K * u .
$$

The method of characteristics is used in many of the analytical arguments to prove the existence and other important properties of the aggregation equation (1), see [12] and [6]. This method provides a natural adaptive grid scheme to concentrate spatial resolution near the blowup point or set, and was employed to investigate gravitational collapse by Brenner and Witelski [14]. Moreover, for nonnegative initial data, we have the monotonicity condition $\frac{\partial}{\partial r} K * u \geq$ $0, \Delta_{r} K * u>0$, i.e., the points always move towards to the origin and the magnitude is always 

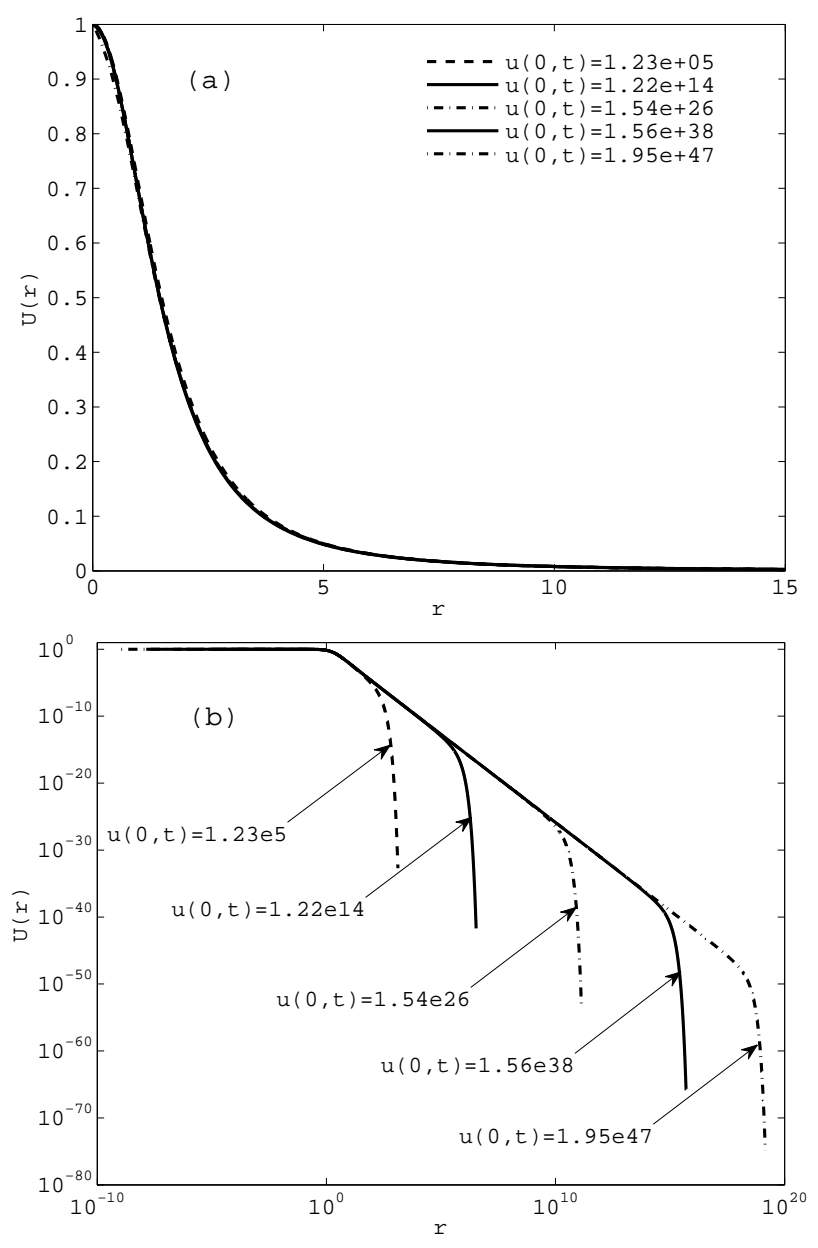

FIG. 2.4. The convergence of the normalized profiles in dimension three. (a) Near the origin, all the profiles are indistinguishable. (b) Far away from the origin, the blowup dynamics adjusts the algebraic decay of the tail.

increasing along the path. Thus our scheme preserves the positivity of the solution. The numerical results indicate that this simple scheme resolves the profiles quite well, both near the core and far away from it. If the self-similarity were of the first kind, then the characteristics would exactly preserve the spatial resolution going into the blowup. Since it is a second-kind similarity solution with anomalous scaling (i.e., the characteristics do not scale in time as the similarity variable) we lose resolution over time, but at a relatively slow rate compared with the dynamics of blowup.

The system (3.2) is solved using the conventional fourth order Runge-Kutta method, with the size of the time step $\Delta t$ adapted according to the following two criteria: (a) The relative increase of the solution at all points is bounded by a threshold at each time step. (b) The nodes cannot cross each other during each time step. Finally, we need to compute the convolution of the kernel. We first give a general formulation for any dimension greater than two and then a special one in odd dimensions three and higher, to reduce computational effort by one order of magnitude.

3.1. General Dimension. Instead of calculating $K * u$ once and taking the numerical derivatives to solve (3.2), we find $\frac{\partial}{\partial r} K * u$ and $\Delta_{r} K * u$ directly by computing the derivatives 

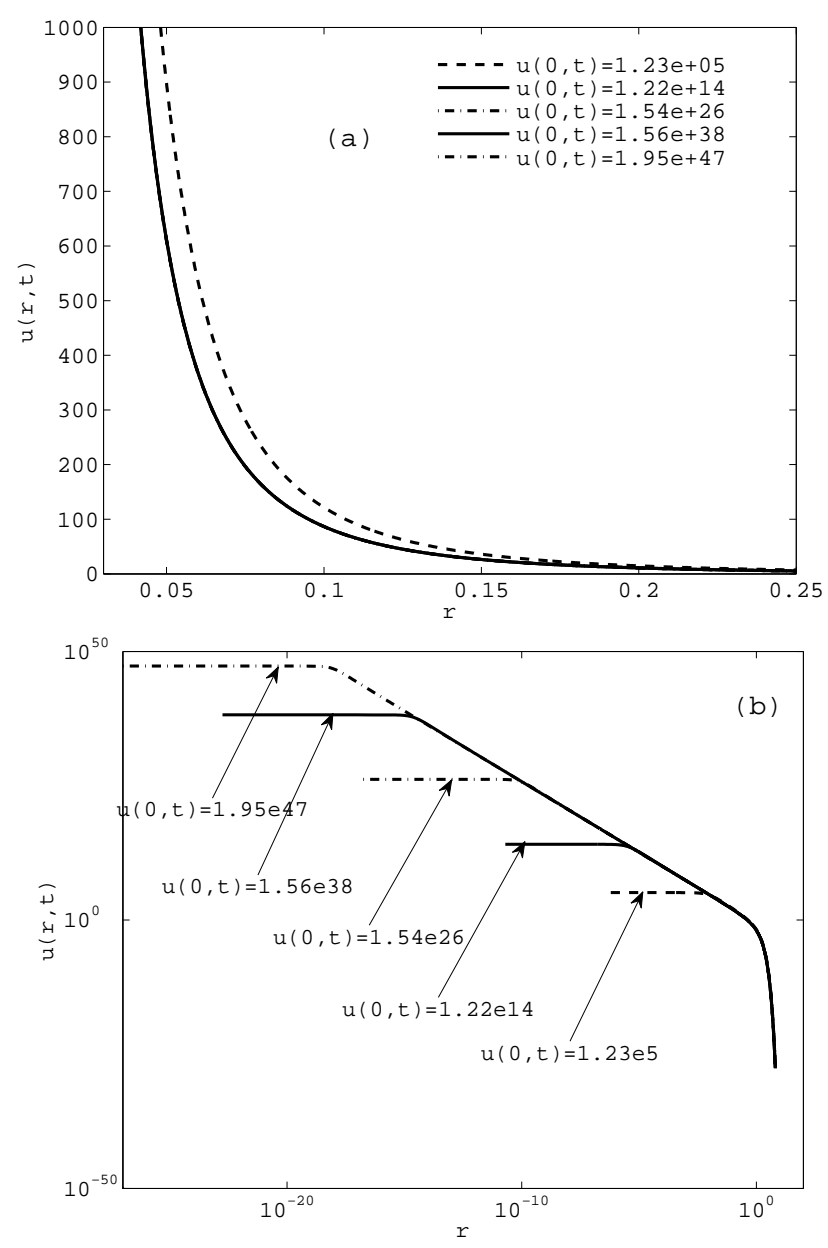

FIG. 2.5. The convergence of the original function $u$ in dimension three. (a) Away from the blowup point, the solution barely changes because the blowup happens in such a short time scale. (b) Close to the blowup point, the solution fills an envelope which becomes infinity at the origin.

of the kernel, i.e.,

$$
\begin{array}{r}
\frac{\partial}{\partial r} K * u=c_{n} \int_{0}^{\infty} u\left(r^{\prime}\right) r^{\prime n-1} \int_{0}^{\pi} \frac{r-r^{\prime} \cos \theta}{\sqrt{r^{2}+r^{\prime 2}-2 r r^{\prime} \cos \theta}} \sin ^{n-2} \theta d \theta d r^{\prime}, \\
\Delta_{r} K * u=(n-1) c_{n} \int_{0}^{\infty} u\left(r^{\prime}\right) r^{\prime n-1} \int_{0}^{\pi} \frac{1}{\sqrt{r^{2}+r^{\prime 2}-2 r r^{\prime} \cos \theta}} \sin ^{n-2} \theta d \theta d r^{\prime},
\end{array}
$$

where $c_{n}$ is the volume of the unit sphere in $R^{n-1}$. The computation can still be expensive, because at each point we have to perform a double integration. The expense can be reduced by observing the homogeneity of the kernel, which gives the following formulation

$$
\begin{gathered}
\int_{0}^{\pi} \frac{r-r^{\prime} \cos \theta}{\sqrt{r^{2}+r^{\prime 2}-2 r r^{\prime} \cos \theta}} \sin ^{n-2} \theta d \theta=\left\{\begin{array}{cc}
\int_{0}^{\pi} \frac{(1-\rho \cos \theta) \sin ^{n-2} \theta}{\sqrt{1+\rho^{2}-2 \rho \cos \theta}} d \theta, & \text { if } r^{\prime} \leq r \\
\int_{0}^{\pi} \frac{(\rho-\cos \theta) \sin ^{n-2} \theta}{\sqrt{1+\rho^{2}-2 \rho \cos \theta}} d \theta, & \text { if } r^{\prime} \geq r
\end{array}\right. \\
\int_{0}^{\pi} \frac{\sin ^{n-2} \theta}{\sqrt{r^{2}+r^{\prime 2}-2 r r^{\prime} \cos \theta}} d \theta=\frac{1}{\max \left(r, r^{\prime}\right)} \int_{0}^{\pi} \frac{\sin ^{n-2} \theta}{\sqrt{1+\rho^{2}-2 \rho \cos \theta}} d \theta,
\end{gathered}
$$


where $\rho=\min \left(r, r^{\prime}\right) / \max \left(r, r^{\prime}\right)$. In this way, the integrations of the kernel with respect to the angular variable have only to be calculated once at the very beginning as functions of $\rho \in[0,1]$, i.e., we only need to perform numerical integrations once for the auxiliary functions

$$
\begin{gathered}
I_{1}(\rho)=\int_{0}^{\pi} \frac{(1-\rho \cos \theta) \sin ^{n-2} \theta}{\sqrt{1+\rho^{2}-2 \rho \cos \theta}} d \theta, \quad I_{2}(\rho)=\int_{0}^{\pi} \frac{(\rho-\cos \theta) \sin ^{n-2} \theta}{\sqrt{1+\rho^{2}-2 \rho \cos \theta}} d \theta, \\
I_{3}(\rho)=\int_{0}^{\pi} \frac{\sin ^{n-2} \theta}{\sqrt{1+\rho^{2}-2 \rho \cos \theta}} d \theta .
\end{gathered}
$$
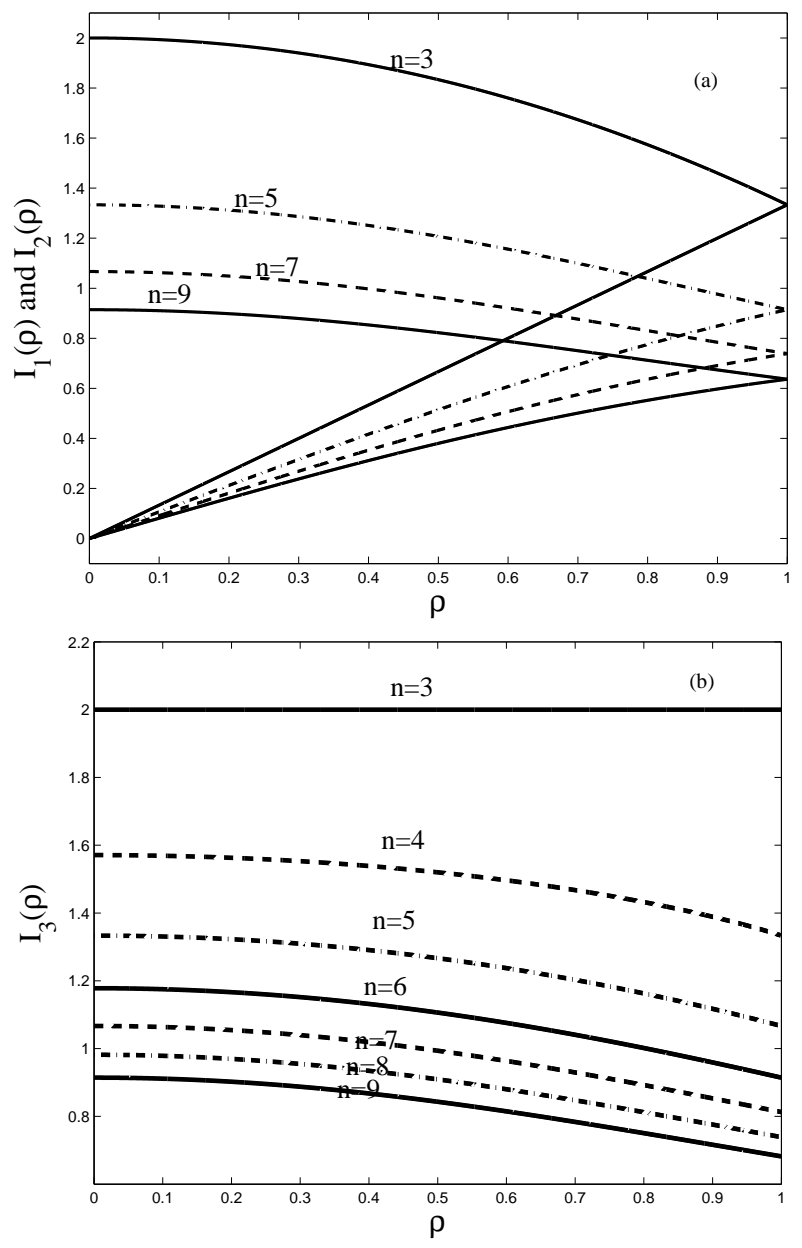

FIG. 3.1. Auxiliary functions in different spatial dimensions: (a) $I_{1}$ (upper branch) and $I_{2}$ (lower branch), (b) $I_{3}$.

The auxiliary variable $\rho$ is chosen such that those integrations are computed only at discrete points and the interpolations of $I_{1}, I_{2}$ and $I_{3}$ are restricted on the bounded interval $[0,1]$. Therefore these functions $I_{1}, I_{2}$ and $I_{3}$ can be computed as accurately as needed without increasing the computation effort during the time evolution. In this way the total computational expense is reduced to $O\left(N^{2}\right)$ at each time step, where $N$ is number of spatial points used to represent the solution. These auxiliary functions (Figure 3.1) are relatively smooth inside the 
interval $[0,1]$ for dimension greater or equal than three. It is easy to see that $I_{3}(1)$ actually becomes divergent as the dimension $n$ less or equal than two. For these reason, the computations are performed only for $n>2$.

3.2. Further reduction in odd dimension. In odd dimension, using the fact that the successive Laplacians of the kernel $K(x)=|x|$ is proportional to the fundamental solution of the Laplace equation, we can further reduce the computation to be $O(N)$ per time step. This is exactly the fact used to prove the nonexistence of mass concentrating self-similar solutions in [7]. First, we start with dimension three to give the basic idea and then generalize it to any odd dimension greater than three. Let $v_{0}=u$, and define $v_{1}$ and $v_{2}$ to be the solutions of the following equations

$$
-\Delta v_{1}=v_{0}, \quad \Delta v_{2}=8 \pi v_{1} \quad \text { in } R^{3},
$$

with $v_{1}$ and $v_{2}$ decay to zero at infinity. We can just write down the solution via the method of fundamental solution, i.e.,

$$
\begin{aligned}
v_{1}(x) & =\int_{R^{3}} \frac{v_{0}(y)}{4 \pi|x-y|} d y \\
v_{2}(x) & =\int_{R^{3}} \frac{2 v_{1}(y)}{|x-y|} d y=\int_{R^{3}} \int_{R^{3}} \frac{v_{0}(z)}{2 \pi|x-y||y-z|} d z d y \\
& =\int_{R^{3}}^{|x-z| u(z) d z=K * u(x) .}
\end{aligned}
$$

In the radial symmetric case, we only need to solve

$$
-\frac{1}{r^{2}} \frac{d}{d r}\left(r^{2} \frac{d v_{1}}{d r}\right)=v_{0}, \quad-\frac{1}{r^{2}} \frac{d}{d r}\left(r^{2} v_{2 r}\right)=8 \pi v_{1},
$$

with the following boundary condition

$$
v_{1}(\infty)=0, \quad \frac{\partial v_{1}(0)}{\partial r}=0, \quad v_{2 r}(0)=0 .
$$

Then the right hand sides of the equations in (3.2) are replaced by

$$
\frac{\partial}{\partial r} K * u=-v_{2 r}, \quad \Delta_{r} K * u=8 \pi v_{1},
$$

with the time scaled by $8 \pi$. Note that we only need to find the derivative $\partial_{r} v_{2}$ of $v_{2}$, instead of $v_{2}$ itself. In actual implementation, the infinity boundary condition $v_{1}(\infty)=0$ is transformed to a condition at $r=0$, i.e., the value of $v_{1}(0)$,

$$
v_{1}(0)=-\int_{0}^{\infty} \frac{\partial v_{1}(r)}{\partial r} d r=\int_{0}^{\infty} \frac{1}{r^{2}} \int_{0}^{r} v_{0}(s) s^{2} d s d r=\int_{0}^{\infty} u(r) r d r .
$$

This integral is usually truncated on a bounded domain if $u$ is compactly supported or decays fast enough. In theory, this transformed boundary condition at the origin gives the unique zero boundary condition at infinity, while any inappropriate choice of $v_{1}$ at the end of the computational domain (an approximation to the condition $v_{1}(\infty)=0$ ) could give a different effective kernel $K$, resulting in some inconsistence in theory and numerics. Once we have the right boundary condition, we can use an $O(N)$ numerical quadrature scheme to find the solution of (3.9), i.e.,

$$
v_{1}(r)=v_{1}(0)-\int_{0}^{r} \frac{1}{\eta^{2}} \int_{0}^{\eta} u(s) s^{2} d s d \tau=v_{1}(0)-\int_{0}^{r} u(s)\left(s-\frac{s^{2}}{r}\right) d s,
$$




$$
v_{2 r}(r)=\frac{8 \pi}{r^{2}} \int_{0}^{r} v_{1}(s) s^{2} d s .
$$

In odd dimension greater than three, with $n=2 k+1$, similarly we introduce $v_{1}, v_{2}, \cdots, v_{k+1}$ such that

$$
-\Delta v_{1}=v_{0},-\Delta v_{2}=v_{1}, \cdots,-\Delta v_{k}=v_{k-1}, \Delta v_{k+1}=d_{k} v_{k} \quad \text { in } R^{n}
$$

and finally set in the characteristics ODEs (3.2)

$$
\frac{\partial}{\partial r} K * u=-\frac{\partial v_{k+1}}{\partial r}, \quad \Delta_{r} K * u=d_{k} v_{k}
$$

where $v_{0}=u$ and

$$
d_{k}=2^{k}(2 k+1) k ! \frac{\pi^{\frac{2 k+1}{2}}}{\Gamma\left(k+1+\frac{1}{2}\right)} .
$$

To transform the boundary condition at infinity to the one at the original, we need to find the appropriate integration like (3.12) with the aid of fundamental solution of the Laplace equation, which is given by

$$
N(x)=\frac{1}{n(n-2) \omega_{n}|x|^{n-2}}, \quad \omega_{n}=\frac{\pi^{n / 2}}{\Gamma(n / 2+1)},
$$

where $\omega_{n}$ is the volume of the unit sphere in $R^{n}$. Using the presentation formula of the solution to the Poisson equation, we have

$$
v_{i}\left(x_{i}\right)=\int_{R^{n}} \cdots \int_{R^{n}} N\left(x_{i}-x_{i-1}\right) N\left(x_{i-1}-x_{i-2}\right) \cdots N\left(x_{1}-x_{0}\right) u\left(x_{0}\right) d x_{0} d x_{1} \cdots d x_{i-1}
$$

for any $1 \leq i \leq k+1$. Translation and rotation invariance of the fundamental solutions gives the following identity

$$
\int_{R^{n}} \cdots \int_{R^{n}} N\left(x_{i}-x_{i-1}\right) N\left(x_{i-1}-x_{i-2}\right) \cdots N\left(x_{1}-x_{0}\right) d x_{1} \cdots d x_{i-1}=N_{i}\left(x_{i}-x_{0}\right),
$$

for some radially symmetric function $N_{i}$. Moreover, dimensional analysis indicates that $N_{i}$ is homogeneous of degree $2 i-n$, i.e.,

$$
N_{i}\left(x_{i}-x_{0}\right)=\frac{c_{i, n}}{n \omega_{n}}\left|x_{i}-x_{0}\right|^{2 i-n}
$$

for some constant $c_{i, n}$. When $i=1$, this is just the fundamental solution, giving the following initial condition

$$
c_{1, n}=\frac{1}{n-2}
$$

We can find a recursive relation for $c_{i, n}$ by taking the negative Laplacian of $N_{i}$ w.r.t $x_{i}$. Formally, on one hand using equation (3.20),

$$
-\Delta_{x_{i}} N_{i}\left(x_{i}-x_{0}\right)=\frac{2(n-2 i)(i-1) c_{i, n}}{n \omega_{n}}\left|x_{i}-x_{0}\right|^{2(i-1)-N} .
$$

On the other hand, using the definition of $N_{i}$,

$$
-\Delta_{x_{i}} \int_{R^{n}} \cdots \int_{R^{n}} N\left(x_{i}-x_{i-1}\right) N\left(x_{i-1}-x_{i-2}\right) \cdots N\left(x_{1}-x_{0}\right) d x_{1} \cdots d x_{i-1}
$$




$$
\begin{aligned}
& =\int_{R^{n}} \cdots \int_{R^{n}} \delta\left(x_{i}-x_{i-1}\right) N\left(x_{i-1}-x_{i-2}\right) \cdots N\left(x_{1}-x_{0}\right) d x_{1} \cdots d x_{i-1} \\
& =\int_{R^{n}} \cdots \int_{R^{n}} N\left(x_{i}-x_{i-2}\right) \cdots N\left(x_{1}-x_{0}\right) d x_{1} \cdots d x_{i-2} \\
& =\frac{c_{i-1, n}}{n \omega_{n}}\left|x_{i}-x_{0}\right|^{2(i-1)-N} .
\end{aligned}
$$

Match the coefficients of above two identities, we have the following recursive formula

$$
c_{i, n}=\frac{1}{2(i-1)(n-2 i)} c_{i-1, n}
$$

and consequently with the initial condition (3.21),

$$
c_{i, n}=\frac{1}{2^{i-1}(i-1) !(n-2 i) ! !},
$$

where $m$ !! is the double factorial of $m$. Finally, we get the boundary condition of $v_{i}$ at the origin in terms of the integral with $u$, i.e.,

$$
v_{i}(0)=\frac{c_{i, n}}{n \omega_{n}} \int_{R^{n}}\left|x_{0}\right|^{2 i-n} u\left(x_{0}\right) d x_{0}=\frac{1}{2^{i-1}(i-1) !(n-2 i) ! !} \int_{0}^{\infty} r^{2 i-1} u(r) d r .
$$

With these boundary conditions, we can find all the auxiliary functions $v_{i}$ s through a series of $O(N)$ numerical integrations like (3.13) to find the right hand side the characteristic ODEs $(3.2)$.

3.3. Numerical Renormalization Group. Since we are more interested in the exponents characterizing the intermediate asymptotics of the dynamics than other quantitative details, we can rescale the solution appropriately to get the the profile. This is the basic principle underlying Renormalization Group Method, which is employed successfully to the numerical investigation of nonlinear filtration and porous medium equations [23, 11].

We start with the solution $u^{(0)}(x, t)=u(x, t)$, whose solution is known on the time interval $\left[t_{0}^{0}, t_{1}^{0}\right]$. Without loss of generality, we let $u^{(0)}\left(0, t_{0}^{0}\right)=1$ and $t_{1}^{0}$ is determined implicitly by $u^{(0)}\left(0, t_{1}^{0}\right)=M$ for some predetermined constant $M>1$. For a given guess of the exponent $\beta_{m}$, at then end of $m$-th iteration, we can renormalize the function as

$$
u^{(m+1)}\left(x, t_{0}^{m+1}\right)=M^{-1} u^{(m)}\left(x M^{-\beta_{m} / \alpha_{m}}, t_{1}^{m}\right), \quad \alpha_{m}=(n-1) \beta_{m}+1 .
$$

An equation for $\beta_{m}$ can be estimated from the spatial-temporal relation of the blowup dynamics. Near blow-up time, we have

$$
u(0, t)=(T-t)^{-\alpha} U_{0}, \quad r_{1 / 2}(t)=(T-t)^{\beta} r_{0},
$$

where $r_{1 / 2}(t)$ is the position where $u$ is half of $u$ at the origin, i.e.,

$$
u\left(r_{1 / 2}(t), t\right)=\frac{1}{2} u(0, t) .
$$

Therefore, on one hand we have

$$
\frac{d \ln u(0, t)}{d \ln r_{1 / 2}(t)}=\frac{d \ln u(0, t) / d t}{d \ln r_{1 / 2}(t) / d t}=-\frac{\alpha}{\beta}=1-n-\frac{1}{\beta} .
$$

On the other hand, using the original evolution equation, we can calculate the time derivatives explicitly, i.e.,

$$
\frac{d \ln u(0, t)}{d \ln r_{1 / 2}(t)}=\frac{r_{1 / 2}(t)}{u(0, t)} \frac{d u(0, t) / d t}{d r_{1 / 2}(t) / d t}=\frac{r_{1 / 2}(t)}{u(0, t)} \frac{\left.\nabla \cdot(u \nabla K * u)\right|_{r=0}}{d r_{1 / 2}(t) / d t}
$$


Finally $d r_{1 / 2}(t) / d t$ can be obtained by taking the time derivative of equation (3.29)

$$
u_{r}\left(r_{1 / 2}(t), t\right) \frac{d r_{1 / 2}(t)}{d t}+u_{t}\left(r_{1 / 2}(t), t\right)=\frac{1}{2} u_{t}(0, t)
$$

or equivalently

$$
\frac{d r_{1 / 2}(t)}{d t}=\frac{\left.\frac{1}{2} \nabla \cdot(u \nabla K * u)\right|_{r=0}-\left.\nabla \cdot(u \nabla K * u)\right|_{r=r_{1 / 2}(t)}}{u_{r}\left(r_{1 / 2}(t), t\right)} .
$$

At the end of $m$-th iteration, the exponent $\beta_{m}$ is solved by combining (3.30) and (3.31), i.e.,

$$
1-n-\frac{1}{\beta_{m}}=\frac{r_{1 / 2}\left(t_{1}^{m}\right)}{u^{(m)}\left(0, t_{1}^{m}\right)} \frac{\left.u_{r}^{(m)}\left(r_{1 / 2}\left(t_{1}^{m}\right), t_{1}^{m}\right) \nabla \cdot\left(u^{(m)} \nabla K * u^{(m)}\right)\right|_{r=0}}{\left.\frac{1}{2} \nabla K * u^{(m)}\right)\left.\right|_{r=0}-\left.\nabla \cdot\left(u^{(m)} \nabla K * u^{(m)}\right)\right|_{r=r_{1 / 2}\left(t_{1}^{m}\right)}}
$$

Above relation is preserved under the renormalization transformation (3.27), in the sense that

$1-n-\frac{1}{\beta_{m}}=\frac{r_{1 / 2}\left(t_{0}^{m+1}\right)}{u^{(m+1)}\left(0, t_{0}^{m}\right)} \frac{\left.u_{r}^{(m+1)}\left(r_{1 / 2}\left(t_{0}^{m+1}\right), t_{0}^{m+1}\right) \nabla \cdot\left(u^{(m+1)} \nabla K * u^{(m+1)}\right)\right|_{r=0}}{\left.\frac{1}{2} \nabla \cdot\left(u^{(m+1)} \nabla K * u^{(m+1)}\right)\right|_{r=0}-\left.\nabla \cdot\left(u^{(m+1)} \nabla K * u^{(m+1)}\right)\right|_{r=r_{1 / 2}\left(t_{0}^{m+1}\right)}}$

Because the renormalized function decays only algebraically even with a compactly supported initial data, the function $u^{(m)}$ is computed on a interval $r \in[0, L]$ and is chosen to be $u(L)(L / r)^{\alpha_{m} / \beta_{m}}$ for $r>L$.

The anomalous exponent $\beta$ computed using this numerical renormalization method is compared with that from direct simulation in Figure 2.3. The former concentrates the computation on the profile and the exponents with a fixed spatial domain while the latter have to resolve the solution on a large spatial domain and eventually cannot give a good fit at lower dimension when the kernel becomes singular. Therefore, the profile and the exponents can be computed with high accuracy without any formation of singularity. On the other hand, the direct simulation tells more details about the blowup dynamics, like various norms of the solution when approaching the blowup time.

For simulation in general dimensions, the auxiliary functions $I_{1}, I_{2}$ and $I_{3}$ are computed on $10^{4}$ equally-spaced points on the interval $[0,1]$. The number of spatial points is 4000 and the whole simulation takes a few days for one single dimension on a $3.0 \mathrm{GHz}$ Intel Pentium IV cluster machine compiled with GNU GCC. For the special formulation in odd dimensions, the number of spatial points is as large as $2 \times 10^{4}$, and the simulation takes usually a few hours. Initially the grid points $\left\{r_{j}\right\}$ are placed such that $\ln \left(1+r_{j}\right)$ is equally spaced on $\left[0, \ln \left(1+r_{N}\right)\right]$. The initial condition is chosen to be Gaussian, even though other smooth, compactly supported functions (not necessary to be radially decreasing) work well too and produce computationally identical similarity solutions. The special code for simulation in odd dimensions gives exponents $\alpha$ and $\beta$ and other parameters consistent with code for general dimensions. The main difference is computational speed. We reiterate that we do not have to perform adaptive mesh refinement because the characteristics due a good job of following the similarity variables, although they are not identical.

4. Additional Numerical Results. Besides those results already shown in previous sections, we gather addition numerical results and verifications to validate the claims made before. 
4.1. Estimation of the exponents. Close to the blowup time, $U(0, \tau)$ should approach a constant $U_{0}$, and $u(0, t) \approx(T-t)^{-\alpha} U_{0}$. The time derivative $u_{t}(0, t)$ can be approximated by $u(0, t)$ too, i.e.,

$$
u_{t}(0, t) \sim \alpha U_{0}^{-1 / \alpha} u(0, t)^{1+1 / \alpha} .
$$

On the other hand, from the second characteristic ODE $(3.2), u_{t}(0, t)=u(0, t) \Delta_{r} K * u(0, t)$, we have

$$
\ln \left(\Delta_{r} K * u(0, t)\right)=\ln \left(\alpha U_{0}^{-1 / \alpha}\right)+\frac{1}{\alpha} \ln u(0, t)
$$

Using $u(0, t), \Delta_{r} K * u(0, t)$ at each time step, a simple least square fitting gives the pair of
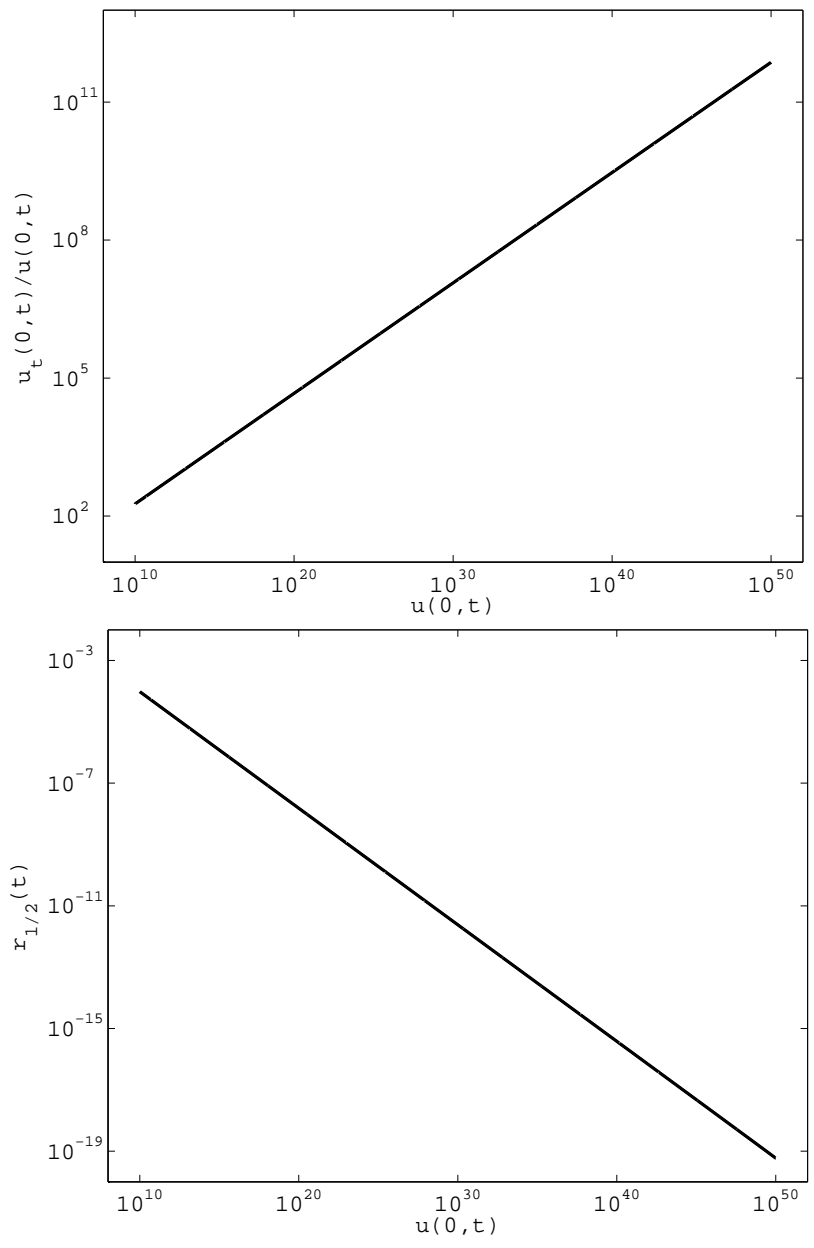

FIG. 4.1. Estimation of $\alpha$ and $\beta$ in three dimension. The straight lines in the log-log plots indicate a strong evidence of the self-similar blowup of the radially symmetric solutions.

parameters $\left(\alpha, U_{0}\right)$. To estimate the exponent $\beta$ for spatial spread, we need to introduce a spatial scale. The most natural one is the half-width of the blowup profile, $r_{1 / 2}(t)$, the position at which the magnitude is half of that at the origin, i.e.,

$$
u\left(r_{1 / 2}(t), t\right)=u(0, t) / 2 .
$$


The similarity form of the blowup implies

$$
r_{1 / 2}(t) \sim r_{0}(T-t)^{\beta}
$$

for some constant $r_{0}$. Using $r_{1 / 2}(t)$ (from interpolation if there is no function value that is exactly half of the maximum magnitude) and $T-t$ estimated with parameters obtained above, we can get $\beta$. In all the parameter estimation, only those data that close to blowup time $\left(u(0, t)>10^{10}\right)$ is used and the profiles should be radially decreasing such that there is one unique $r_{1 / 2}(t)$. The simulation is terminated when $u(0, t)$ reaches an upper bound $10^{50}$ provided that the profile near the origin is well resolved, say there are at least one hundred points nodes on the interval $\left[0, r_{1 / 2}(t)\right]$.

4.2. Blowup in $L^{p}$ norm. Since the blow up does not concentrate any mass, it is possible that the $L^{p}$ norm of the solution could still be finite for some $p>1$ at the blowup time. Using the self-similar form verified in previous sections, we can give a more quantitative characterization of the blow up in $L^{p}$ norm. First, we have

$$
\|u\|_{L^{p}}^{p}=\int_{R^{n}} u^{p} d x=(T-t)^{-\alpha p+n \beta} \int_{R^{n}} U^{p} d y=(T-t)^{-\alpha p+n \beta} C \int_{0}^{\infty} U^{p} r^{n-1} d r .
$$

Let the critical exponent be defined as $p^{*}=n \beta / \alpha$. To the leading order, $U$ has an algebraic decay rate of the form $r^{-\alpha / \beta}$, and this rate of decay is extended to infinity as $t \rightarrow T$. If $p>p^{*}$, the integrand $U^{p} r^{n-1}$ decays fast enough and the last integral in equation(4.5) is uniformly bounded and nonzero, therefore the behavior of $\|u\|_{L^{p}}$ is determined by $(T-t)^{-\alpha\left(1-p^{*} / p\right)}$, which becomes infinity.

For $p \in\left(1, p^{*}\right)$, the integral in the last expression blows up but the factor $(T-t)^{-\alpha p+n \beta}$ goes to zero. Therefore, we need a more delicate estimate for the integral using the fact that $U$ has an algebraic decay only up to some large distance $R(t)$. This upper bound can be estimated from the total mass for $U$. On one hand, from the evolution equation for $U$, we have,

$$
\frac{d}{d \tau} \int_{R^{n}} U(y, \tau) d y=(\beta-1) \int_{R^{n}} U(y, \tau) d y
$$

or

$$
\int_{R^{n}} U(y, \tau) d y=e^{(\beta-1) \tau}\left[e^{(\beta-1) \ln T} \int_{R^{n}} U(y,-\ln T) d y\right]=(T-t)^{-(\beta-1)} M_{0},
$$

where $M_{0}$ is a constant depending only on the initial condition. On the other hand, the integral above can be approximated by assuming $U(y, \tau)$ has an algebraic decay of order $O\left(|y|^{-\alpha / \beta}\right)$ exactly up to $R(t)$ and is zero beyond $R(t)$, i.e., for $t$ close to the blowup time $T$,

$$
\int_{R^{n}} U(y, \tau) d y \approx \int_{R_{0}}^{R(t)} C r^{-\alpha / \beta+n-1} d r \approx C_{1} R(t)^{n-\alpha / \beta}=C_{1} R(t)^{1-1 / \beta} .
$$

Compared with (4.7),

$$
R(t) \approx C_{2}(T-t)^{-\beta}
$$

and consequently the $L^{p}$ norm is

$$
\|u\|_{L^{p}}^{p} \approx C(T-t)^{-\alpha p+n \beta} \int_{0}^{R(t)} r^{-\alpha p / \beta+n-1} d r=C_{1}(T-t)^{-\alpha p+n \beta-\beta(-\alpha p / \beta+n)}=C_{1} .
$$

In another word, if $p \in\left(1, p^{*}\right),\|u\|_{L^{p}}$ is still uniformly bounded up to blowup time. 


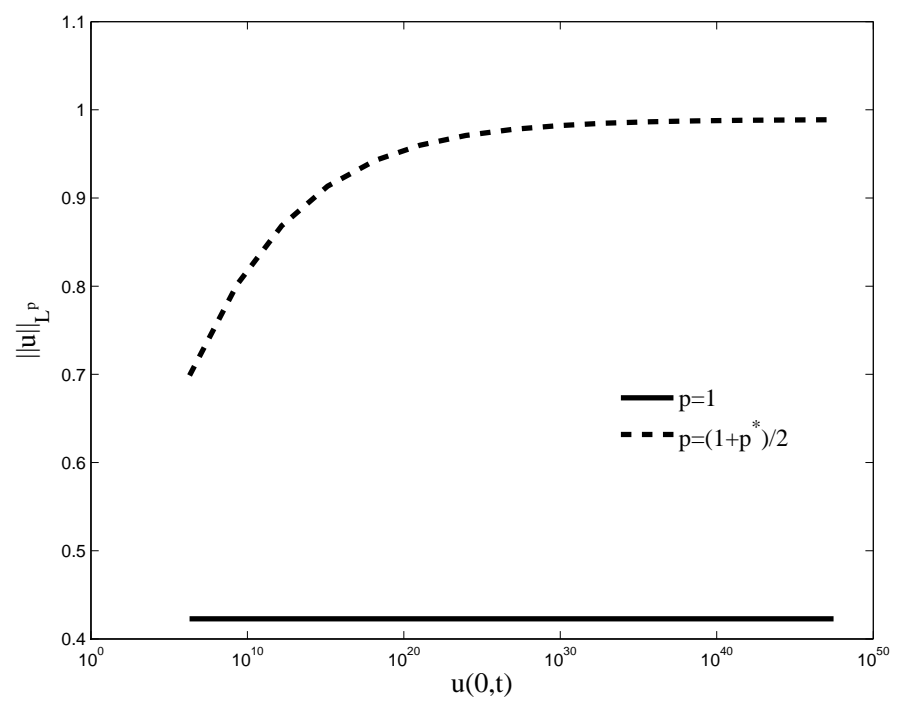

FIG. 4.2. $\|u\|_{L^{p}}$ for $p=1$ and $p=\left(1+p^{*}\right) / 2\left(<p^{*}\right)$ in dimension $n=3$. Note that the mass $(p=1)$ is perfectly conserved in our numerical scheme.

At the critical norm $p=p^{*}$,

$$
\|u\|_{L^{p^{*}}}^{p^{*}} \approx C \int_{R_{0}}^{R(t)} r^{-1} d r=C_{1}+C_{2} \ln R(t)=C_{1}-C_{2} \beta \ln (T-t) .
$$

For all three cases, the above analysis is in perfect agreement with numerical observation of the norms (Figure 4.2 and 4.3). The total mass $\|u\|_{L^{1}}$ is still perfectly conserved even for $u(0, t)$ close to $10^{50}$. In the critical $\left(p=p^{*}\right)$ and supercritical $\left(p>p^{*}\right)$ case, the blowup of the norms fit the expected form of logarithm and power law blowup very well. This blowup result is also consistent with the $L^{p}$ theory developed in a companion paper [10], in which the local well-posedness of the equation is proved for initial data in $L^{p}$ space with $p$ greater than $p_{s}=n /(n-1)>p^{*}$. At the time of blowup, our numerical solution exhibits a pure power law behavior at the origin. in [10] it is shown that continuation of a solution of this type leads to instantaneous mass concentration, hence proving that the dynamics is ill-posed in the $L^{p}$ for sufficiently small $p$. Thus the numerical results here, combined with the analysis of that paper, give a complete picture of the dynamics, from smooth initial data, to finite time blowup with a power law singularity, and then instantaneous mass concentration after the initial singularity. We note that this is a multidimensional generalization of how singularities form in Burgers equation, which we have shown is equivalent to our problem in one dimension. In the Burgers case, the initial singularity for equation (2.10) involves $\phi(x, T) \sim x^{1 / 3}$ at the blowup time, which, following the classical theory of conservation laws, instantaneously leads to shock formation with a jump discontinuity, corresponding to mass concentration in the corresponding aggregation problem. Thus we see the same phenomena occurring in multiple dimensions.

5. Conclusions. We have studied the blowup behavior of solutions of the aggregation equation $u_{t}=\nabla \cdot(u \nabla K * u)$ in multiple dimensions for the kernel $K(x)=|x|$. The numerical observations give strong evidence that the solutions are self-similar and of the second kind. Even though the numerical results are consistent with known theory, in particular the lack of first kind similarity solutions in odd dimensions higher than one [7], there are still many important questions left behind. First, the solutions are computed only in the radially symmetric case. However, this radial symmetry could be broken by a non-radially symmetric perturbation. We 

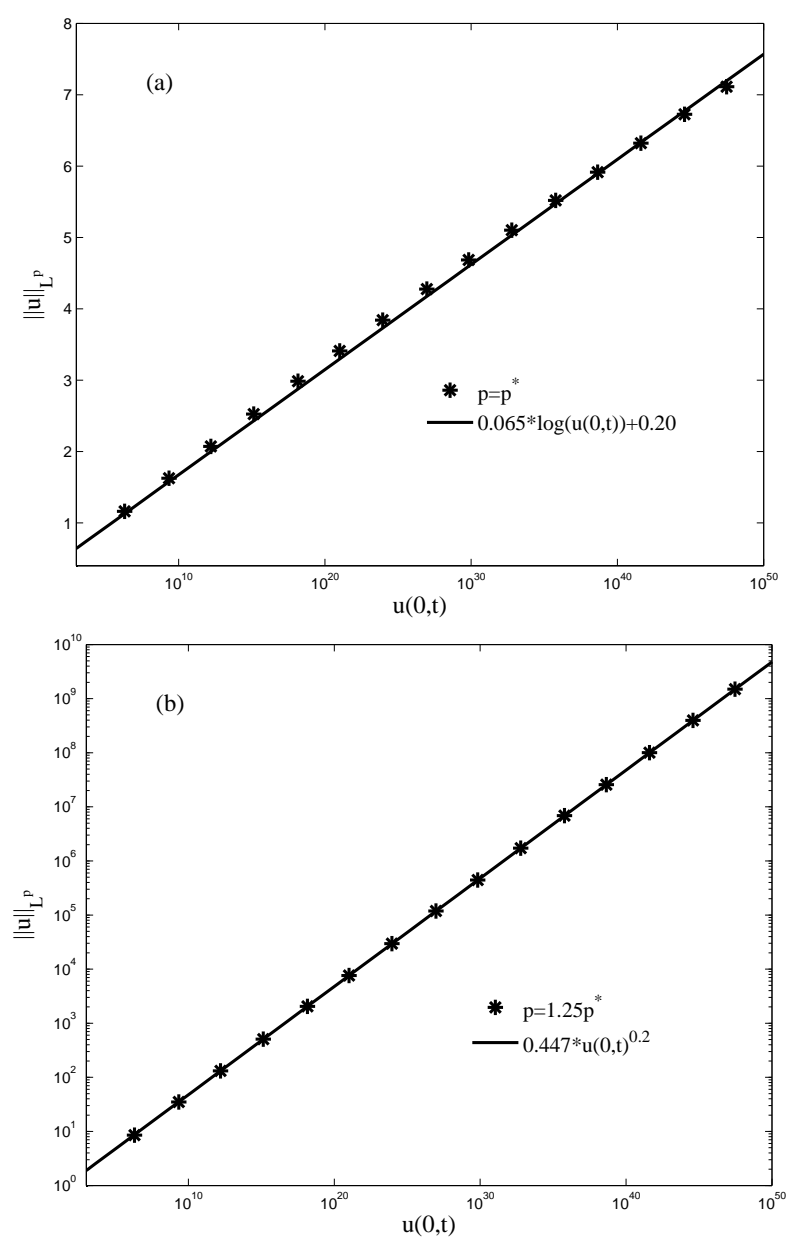

FIG. 4.3. Logarithmic blowup of $\|u\|_{L^{p}}$ for $p=p^{*}(a)$ and power law blow up for $p>p^{*}(b)$ in dimension $n=3$. In the latter case $1 / 5$ is exactly the theoretic predicted value $1-p^{*} / p$.

have not performed linear stability theory for this problem, as has been done in other examples of self-similarity [5] and this would be interesting, both for the radially symmetric and non-radial cases. Likewise, we have not done a systematic study of solutions of the similarity equation (2.7) - as has been shown for other problems $[26,50]$ there may exist unstable similarity solutions with different shape from the ones found in this paper. Moreover, the homogeneity of the kernel can be generalized to $K(x)=|x|^{\gamma}$, which has been proposed for some one dimensional models of granular flow [21, 41] and the dynamics of blowup for such kernels is an interesting open problem in multiple dimensions. We also note that there are recent theoretical results on blowup for this class of problems with additional linear and nonlinear diffusion [39, 40] and it would be interesting to understand blowup profiles for these problems as well. Our method of characteristics does not directly apply to such problems due to the diffusive nature of the dynamics. One possibility, which we have not discussed here, is to use ideas from optimal transport theory, which applies to this class of equations [7]. A recent numerical scheme built on this idea, for diffusive and aggregation phenomena, has been introduced in [22].

Acknowledgments. This work is supported by NSF grant DMS-0907931 and ONR grant N000140710431. We thank Thomas Laurent for helpful suggestions and Thomas P. Witelski 
for comments on an early draft.

\section{REFERENCES}

[1] D. G. Aronson and J. Graveleau. A selfsimilar solution to the focusing problem for the porous medium equation. European J. Appl. Math., 4:65-81, 1993.

[2] D. G. Aronson and J. L. Vázquez. Anomalous exponents in nonlinear diffusion. J. Nonlinear Sci., 5(1):2956, 1995.

[3] G. I. Barenblatt and G. I. Sivashinskii. Self-similar solutions of the second kind in nonlinear filtration. J. Appl. Math. Mech., 33:836-845 (1970), 1969.

[4] Grigory Isaakovich Barenblatt. Scaling, self-similarity, and intermediate asymptotics, volume 14 of Cambridge Texts in Applied Mathematics. Cambridge University Press, Cambridge, 1996. With a foreword by Ya. B. Zeldovich.

[5] Andrew J. Bernoff, Andrea L. Bertozzi, and Thomas P. Witelski. Axisymmetric surface diffusion: dynamics and stability of self-similar pinchoff. J. Statist. Phys., 93(3-4):725-776, 1998.

[6] Andrea L. Bertozzi and Jeremy Brandman. Finite-time blow-up of $L^{\infty}$-weak solutions of an aggregation equation, 2008. accepted in Communications in the Mathematical Sciences.

[7] Andrea L. Bertozzi, Jose A. Carrillo, and Thomas Laurent. Blow-up in multidimensional aggregation equations with mildly singular interaction kernels. Nonlinearity, 22(3):683-710, 2009.

[8] Andrea L. Bertozzi and Thomas Laurent. Finite-time blow-up of solutions of an aggregation equation in $\mathbf{R}^{n}$. Comm. Math. Phys., 274(3):717-735, 2007.

[9] Andrea L. Bertozzi and Thomas Laurent. The behavior of solutions of multidimensional aggregation equations with mildly singular interaction kernels. Chinese Annals of Mathematics, Series B, 2009. (in press).

[10] Andrea L. Bertozzi, Thomas Laurent, and J Rosado. $L^{p}$ theory for the multidimensional aggregation equation, 2009.

[11] S. I. Betelú, D. G. Aronson, and S. B. Angenent. Renormalization study of two-dimensional convergent solutions of the porous medium equation. Phys. D, 138(3-4):344-359, 2000.

[12] M. Bodnar and J. J. L. Velazquez. An integro-differential equation arising as a limit of individual cell-based models. J. Differential Equations, 222(2):341-380, 2006.

[13] M. P Brenner, P. Constantin, L P. Kadanoff, A. Schenkel, and S. C.Venkataramani. Diffusion, attraction and collapse. Nonlinearity, 12(4):1071-1098, 1999.

[14] Michael P. Brenner and Thomas P. Witelski. On spherically symmetric gravitational collapse. J. Statist. Phys., 93(3-4):863-899, 1998.

[15] C. J. Budd, R. Carretero-González, and R. D. Russell. Precise computations of chemotactic collapse using moving mesh methods. J. Comput. Phys., 202(2):463-487, 2005.

[16] C. J. Budd, V. A. Galaktionov, and J. F. Williams. Self-similar blow-up in higher-order semilinear parabolic equations. SIAM J. Appl. Math., 64(5):1775-1809 (electronic), 2004.

[17] Chris Budd and Victor Galaktionov. Stability and spectra of blow-up in problems with quasi-linear gradient diffusivity. R. Soc. Lond. Proc. Ser. A Math. Phys. Eng. Sci., 454(1977):2371-2407, 1998.

[18] Chris J. Budd, Weizhang Huang, and Robert D. Russell. Moving mesh methods for problems with blow-up. SIAM J. Sci. Comput., 17(2):305-327, 1996.

[19] Martin Burger, Vincenzo Capasso, and Daniela Morale. On an aggregation model with long and short range interactions. Nonlinear Anal. Real World Appl., 8(3):939-958, 2007.

[20] Martin Burger and Marco Di Francesco. Large time behavior of nonlocal aggregation models with nonlinear diffusion. Networks and Heterogeneous Media (NHM), 3(4):749 - 785, 2008.

[21] E. Caglioti and C. Villani. Homogeneous cooling states are not always good approximations to granular flows. Arch. Ration. Mech. Anal., 163(4):329-343, 2002.

[22] J. A. Carrillo and J. S. Moll. Numerical simulation of diffusive and aggregation phonemena in nonlinear continuity equations by evolving diffeomorphisms, 2008. preprint submitted.

[23] L.Y. Chen and N. Goldenfeld. Numerical renormalization-group calculations for similarity solutions and traveling waves. Physical Review E, 51(6):5577-5581, 1995.

[24] R. Y. Chiao, E. Garmire, and C. H. Townes. Self-trapping of optical beams. Phys. Rev. Lett., 13(15):479482, Oct 1964.

[25] J. W. Dold. Analysis of the early stage of thermal runaway. The Quarterly Journal of Mechanics and Applied Mathematics, 38(3):361-387, 1985.

[26] J. Eggers and M. A. Fontelos. The role of self-similarity in singularities of PDE's. Nonlinearity, 22(1):R1, 2009.

[27] Gadi Fibich, Nir Gavish, and Xiao-Ping Wang. New singular solutions of the nonlinear Schrödinger equation. Phys. D, 211(3-4):193-220, 2005.

[28] Stathis Filippas and Robert V. Kohn. Refined asymptotics for the blowup of $u_{t}-\Delta u=u^{p}$. Comm. Pure Appl. Math., 45(7):821-869, 1992.

[29] Yoshikazu Giga and Robert V. Kohn. Asymptotically self-similar blow-up of semilinear heat equations. 
Comm. Pure Appl. Math., 38(3):297-319, 1985.

[30] R. T. Glassey. On the blowing up of solutions to the Cauchy problem for nonlinear Schrodinger equations. Journal of Mathematical Physics, 18(9):1794-1797, 1977.

[31] Nigel Goldenfeld, Olivier Martin, Y. Oono, and Fong Liu. Anomalous dimensions and the renormalization group in a nonlinear diffusion process. Phys. Rev. Lett., 64(12):1361-1364, Mar 1990.

[32] Darryl D. Holm and Vakhtang Putkaradze. Aggregation of finite-size particles with variable mobility. Phys Rev Lett, 95:226106, 2005.

[33] Darryl D. Holm and Vakhtang Putkaradze. Formation of clumps and patches in self-aggregation of finitesize particles. Phys. D, 220(2):183-196, 2006.

[34] Weizhang Huang, Jingtang Ma, and Robert D. Russell. A study of moving mesh PDE methods for numerical simulation of blowup in reaction diffusion equations. J. Comput. Phys., 227(13):6532-6552, 2008.

[35] Josephus Hulshof and Juan Luis Vázquez. Self-similar solutions of the second kind for the modified porous medium equation. European J. Appl. Math., 5(3):391-403, 1994.

[36] S. Kamin, L. A. Peletier, and J. L. Vázquez. On the Barenblatt equation of elastoplastic filtration. Indiana Univ. Math. J., 40(4):1333-1362, 1991.

[37] E.F. Keller and L.A. Segel. Initiation of slime mold aggregation viewed as an instability. J. Theor. Biol., 26:399415, 1970.

[38] Thomas Laurent. Local and global existence for an aggregation equation. Comm. Partial Differential Equations, 32(10-12):1941-1964, 2007.

[39] D. Li and J. Rodrigo. Finite-time singularities of an aggregation equation in $R^{n}$ with fractional dissipation. Comm. Math. Phys., 287(2):687-703, 2009.

[40] D. Li and J. Rodrigo. Refined blowup criteria and nonsymmetric blowup of an aggregation equation. Adv. Math., 220(6):1717-1738, 2009.

[41] Hailiang Li and Giuseppe Toscani. Long-time asymptotics of kinetic models of granular flows. Arch. Ration. Mech. Anal., 172(3):407-428, 2004.

[42] D. W. McLaughlin, G. C. Papanicolaou, C. Sulem, and P. L. Sulem. Focusing singularity of the cubic Schrödinger equation. Phys. Rev. A, 34(2):1200-1210, Aug 1986.

[43] Frank Merle and Hatem Zaag. Stability of the blow-up profile for equations of the type $u_{t}=\Delta u+|u|^{p-1} u$. Duke Math. J., 86(1):143-195, 1997.

[44] Alexander Mogilner and Leah Edelstein-Keshet. A non-local model for a swarm. J. Math. Biol., 38(6):534570,1999

[45] K. D. Moll, Alexander L. Gaeta, and Gadi Fibich. Self-similar optical wave collapse: Observation of the townes profile. Phys. Rev. Lett., 90(20):203902, May 2003.

[46] L. A. Peletier. The anomalous exponent of the Barenblatt equation. European J. Appl. Math., 5(2):165175,1994

[47] A A. Samarskii, V A. Galaktionov, S P. Kurdyumov, and A P. Mikhailov. Blow-up in quasilinear parabolic equations, volume 19. Walter de Gruyter \& Co., Berlin, 1995.

[48] Chad M. Topaz and Andrea L. Bertozzi. Swarming patterns in a two-dimensional kinematic model for biological groups. SIAM J. Appl. Math., 65(1):152-174 (electronic), 2004.

[49] Chad M. Topaz, Andrea L. Bertozzi, and Mark A. Lewis. A nonlocal continuum model for biological aggregation. Bull. Math. Biol., 68(7):1601-1623, 2006.

[50] T. P. Witelski, A. J. Bernoff, and A. L. Bertozzi. Blowup and dissipation in a critical-case unstable thin film equation. European J. Appl. Math., 15(2):223-256, 2004. 